\title{
Delivery and reveal of localization of upconversion luminescent microparticles and quantum dots in the skin in vivo by fractional laser microablation, multimodal imaging, and optical clearing
}

Elena K. Volkova

Irina Yu Yanina

Elina A. Genina

Alexey N. Bashkatov

Julia G. Konyukhova

Alexey P. Popov

Elena S. Speranskaya

Alla B. Bucharskaya

Nikita A. Navolokin

Irina Yu. Goryacheva

Vyacheslav I. Kochubey

Gleb B. Sukhorukov

Igor V. Meglinski

Valery V. Tuchin 


\title{
Delivery and reveal of localization of upconversion luminescent microparticles and quantum dots in the skin in vivo by fractional laser microablation, multimodal imaging, and optical clearing
}

\author{
Elena K. Volkova, ${ }^{a, b, c, \star}$ Irina Yu Yanina, ${ }^{a, c}$ Elina A. Genina, ${ }^{a, c}$ Alexey N. Bashkatov, ${ }^{a, c}$ Julia G. Konyukhova, ${ }^{a}$ \\ Alexey P. Popov, ${ }^{b, c, d}$ Elena S. Speranskaya, ${ }^{e}$ Alla B. Bucharskaya, ${ }^{,}$Nikita A. Navolokin, ${ }^{f}$ Irina Yu. Goryacheva, ${ }^{e}$ \\ Vyacheslav I. Kochubey, ${ }^{a, b}$ Gleb B. Sukhorukov, ${ }^{a, g}$ Igor V. Meglinski, ${ }^{b, c, h}$ and Valery V. Tuchin ${ }^{a, c, i, j}$ \\ a Saratov State University (National Research University), Optics and Biophotonics Department, Saratov, Russia \\ 'University of Oulu, Optoelectronics and Measurement Techniques Research Unit, Oulu, Finland \\ 'Tomsk State University (National Research University), Interdisciplinary Laboratory of Biophotonics, Tomsk, Russia \\ ITMO University, Terahertz Biomedicine Laboratory, St. Petersburg, Russia \\ eSaratov State University (National Research University), General and Inorganic Chemistry Department, Saratov, Russia \\ fSaratov State Medical University, Saratov, Russia \\ פQueen Mary University of London, School of Engineering and Materials Science, London, United Kingdom \\ hIrkutsk State University, Institute of Biology, Irkutsk, Russia \\ 'ITMO University, Laboratory of Femtomedicine, St. Petersburg, Russia \\ Institute of Precision Mechanics and Control of the Russian Academy of Sciences, Laboratory of Laser Diagnostics of Technical and \\ Living Systems, Saratov, Russia
}

\begin{abstract}
Delivery and spatial localization of upconversion luminescent microparticles $\left[\mathrm{Y}_{2} \mathrm{O}_{3}: \mathrm{Yb}, \mathrm{Er}\right]$ (mean size $\sim 1.6 \mu \mathrm{m})$ and quantum dots (QDs) (CulnS $2 / \mathrm{ZnS}$ nanoparticles coated with polyethylene glycol-based amphiphilic polymer, mean size $\sim 20 \mathrm{~nm}$ ) inside rat skin was studied in vivo using a multimodal optical imaging approach. The particles were embedded into the skin dermis to the depth from 300 to $500 \mu \mathrm{m}$ through microchannels performed by fractional laser microablation. Low-frequency ultrasound was applied to enhance penetration of the particles into the skin. Visualization of the particles was revealed using a combination of luminescent spectroscopy, optical coherence tomography, confocal microscopy, and histochemical analysis. Optical clearing was used to enhance the image contrast of the luminescent signal from the particles. It was demonstrated that the penetration depth of particles depends on their size, resulting in a different detection time interval (days) of the luminescent signal from microparticles and QDs inside the rat skin in vivo. We show that luminescent signal from the upconversion microparticles and QDs was detected after the particle delivery into the rat skin in vivo during eighth and fourth days, respectively. We hypothesize that the upconversion microparticles have created a long-time depot localized in the laser-created channels, as the QDs spread over the surrounding tissues. ๑ 2018 Society of Photo-Optical Instrumentation Engineers (SPIE) [DOI: 10.1117/1.JBO.23.2.026001]
\end{abstract}

Keywords: upconversion microparticles; quantum dots; skin; fractional laser microablation; optical clearing; luminescence spectroscopy; optical coherence tomography; confocal microscopy; histochemical analysis.

Paper 170580PR received Aug. 31, 2017; accepted for publication Jan. 9, 2018; published online Feb. 5, 2018.

\section{Introduction}

Transcutaneous delivery of micro- and nanocarriers directly to the targeted sites inside skin allows for excluding the interaction of drugs with the surrounding tissue during the diffusion and creating a long-time drug depot inside the skin. Passive diffusion of the micro- and nanoparticles into deeper layers of skin is extremely hampered due to protective properties of the skin barrier. ${ }^{1}$ Both size and formulation of the particles govern their penetration into skin. ${ }^{2}$ For example, less than $0.03 \%$ of the applied $\mathrm{ZnO}$ nanoparticles with an average size $\leq 100 \mathrm{~nm}$ included in a sunscreen formulation penetrate to the human epidermis. ${ }^{3}$ As it has been shown by many authors, at skin topical application, the main site of nanoparticle localization in the skin is hair follicles. ${ }^{2,4}$ In particular, in Ref. 4, the authors

*Address all correspondence to: Elena K. Volkova, E-mail: volkovae87@gmail .com have studied cutaneous localization of nonbiodegradable polystyrene nanoparticles labeled by fluorescein 5-isothiocyanate using confocal laser scanning microscopy (CLSM). It follows from the CLSM images that accumulation of 20- and 200-nm polystyrene particles in follicular openings depends on both size of the particles and time elapsed, and there are no evidences of permeation of the nanoparticles in the nonfollicular region. The authors of Ref. 5 also demonstrate accumulation of $\mathrm{ZnO}$ and $\mathrm{TiO}_{2}$ nanoparticles in hair follicles. To implement targeted particle delivery deep into the skin, massage, ${ }^{6}$ low-frequency sonophoresis, ${ }^{7,8}$ microporation, ${ }^{9}$ and fractional laser microablation (FLMA) ${ }^{10,11}$ are typically used. FLMA in combination with ultrasonic treatment is a promising technique for this purpose. ${ }^{12}$

Creating artificial microchannels in the upper skin layers by FLMA promotes deeper and more efficient delivery of microand nanoparticles. The sufficiently fast restoration of the skin

$1083-3668 / 2018 / \$ 25.00$ @ 2018 SPIE 
barrier integrity occurring after particle administration reduces the risk of skin infection. ${ }^{13}$ Ultrasonic treatment facilitates administration of micro- and nanoparticles into the skin and their targeted subsurface localization within the tissue. ${ }^{12}$

One of the most rapidly developing applications of luminescent particles is their use in molecular and cellular biology, medical diagnostics and therapy. ${ }^{14-17}$ For example, in order to monitor encapsulated drug delivery pathways, luminescent particles are introduced into microcapsules at the stage of synthesis. $^{18,19}$

Currently, quantum dots (QDs) and upconversion particles (UCPs), along with the traditionally used luminescent labels, are widely used for biological research. ${ }^{20-25}$ The use of luminescent spectroscopy allows for detection of optical signal from micro- and nanoparticles in real time during several days. ${ }^{26}$ In Ref. 22, the authors have studied the transdermal delivery capacity of water-soluble CdSeS QDs through mouse skin and their deposition in the body. Experimental results indicate that QDs can penetrate into the dermal layer through hair follicles and then get into blood circulation. QDs were found in liver and kidney. Dye-containing polymeric 320-nm nanoparticles accumulated in the hair follicles can stay there for up to 10 days, while a nonparticulate form can be detected only up to 4 days. ${ }^{6}$ Subcutaneous injection of ethylenediaminetetra (methylenephosphonic acid)-coated $(28.9 \pm 1.0 \mathrm{~nm})$ and uncoated $(39.2 \pm 0.3 \mathrm{~nm}) \quad \mathrm{NaYF}_{4}: \mathrm{Er} / \mathrm{Yb}$ nanoparticles in mice has demonstrated that the particles of both types exhibit bright green luminescence upon laser excitation of the skin for up to three days after injection. ${ }^{21}$

In order to visualize spatial localization of the luminescent nanoprobe in tissues, deep penetration of excitation radiation into the tissue is required. In addition, the emitted optical radiation has to be in the so-called "diagnostic" spectral window. ${ }^{27}$ For this, the upconversion luminescence excited in the nearinfrared (NIR) spectral range can be effectively used. Indeed, high scattering of the visible and NIR radiation in tissues as well as optical detection method significantly limits the spatial resolution and the probing depth. ${ }^{27,28}$ This problem can be solved by application of hyperosmotic immersion liquids known as optical clearing agents (OCAs). ${ }^{20,29}$

The combined use of several optical imaging techniques, so called "multimodal imaging," can provide more advanced opportunities to visualize spatial distribution of micro- and nanoparticles within tissues.

In this paper, multimodal optical imaging, i.e., luminescent spectroscopy, optical coherence tomography and confocal microscopy, and immersion optical clearing technique are used for spatial localization and monitoring of upconversion luminescent microparticles and QDs during days within rat skin microchannels created by FLMA.

\section{Materials and Methods}

\subsection{Animals}

In vivo studies were carried out on six outbred albino rats 1 year of age and the body mass of $\sim 400 \mathrm{~g}$. During all experimental studies, animals were kept in accordance with the European Convention for the Protection of animals used for experimental and other scientific purposes. ${ }^{30}$ Prior to all treatments, the rats were anesthetized with Zoletil 50 (Virbac, France) in a dose of $0.05 \mathrm{mg} / \mathrm{kg}$. On the sites under study, hair was thoroughly removed using a cream for depilation.

\subsection{Particles}

Commercially available lanthanide-doped UCPs $\left[\mathrm{Y}_{2} \mathrm{O}_{3}: \mathrm{Yb}, \mathrm{Er}\right]$ PTIR660-UF (Phosphor Technology, United Kingdom) with an average size of $\sim 1.6 \mu \mathrm{m}$ and in-house synthesized $\mathrm{CuInS}_{2} / \mathrm{ZnS}$ QDs with an average size $\sim 20$-nm coated with polyethylene glycol (PEG)-based amphiphilic polymer (see Appendix) were used in the experiments.

The UCPs have a luminescent band near the 650-nm wavelength, upon 980-nm laser excitation [Fig. 1(a)]. The UCPs $\left[\mathrm{Y}_{2} \mathrm{O}_{3}: \mathrm{Yb}, \mathrm{Er}\right]$ are a promising new generation of agents for bioimaging. Upconversion as a phenomenon utilizes

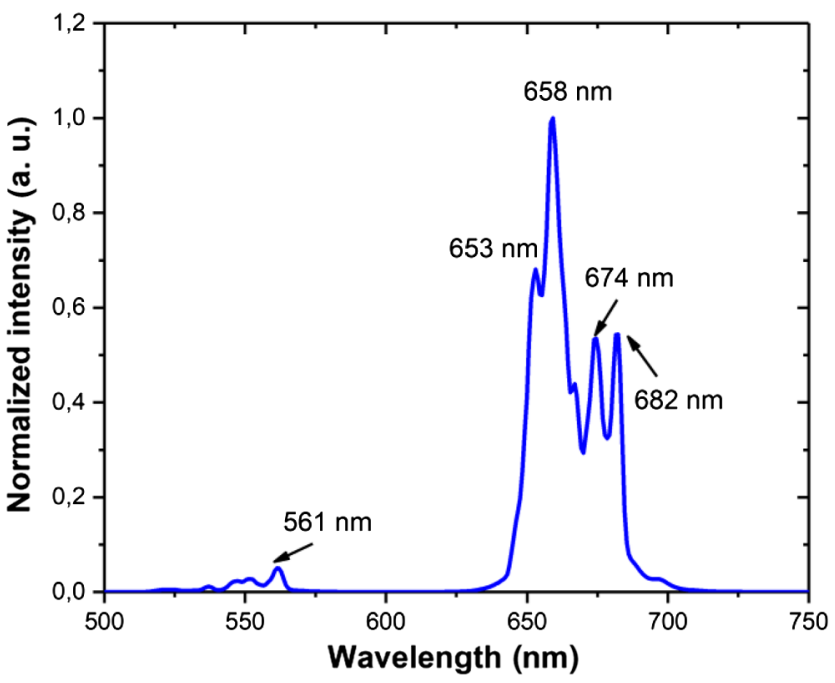

(a)

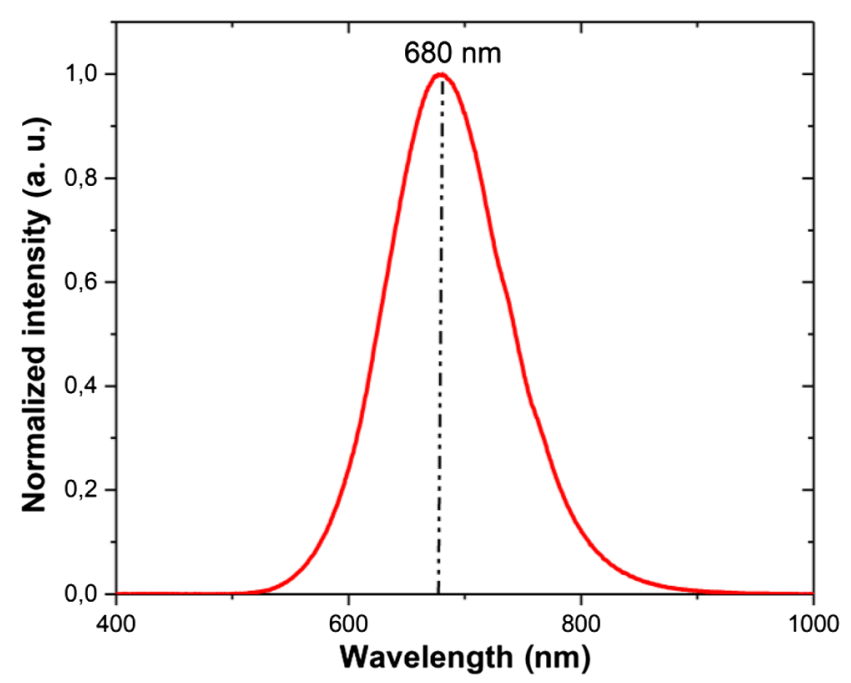

(b)

Fig. 1 Luminescent spectra of (a) UCP (excitation wavelength $980 \mathrm{~nm}$ ) and (b) QDs (excitation wavelength $405 \mathrm{~nm}$ ). 
a sequential absorption of multiple photons via long lifetime (metastable) and real ladder-like energy levels of trivalent lanthanide ions embedded in an appropriate inorganic host lattice to produce higher energy anti-Stokes luminescence. ${ }^{31-33}$ It thereby converts two or more low-energy excitation photons of NIR light into shorter wavelength emission in NIR and visible ranges. High photostability, large blue shift of the upconversion luminescence relative to the wavelength of the exciting radiation, and absorption in the range of maximal transparency of tissues (600 to $1000 \mathrm{~nm}$ ) make it possible to track single particles and perform their long-term detection. ${ }^{34}$

The synthesized QDs have an irregular shape with the crystal size less than $10 \mathrm{~nm}$ and a hydrodynamic diameter around $20 \mathrm{~nm} .{ }^{35}$ The luminescent peak of QDs is at the 680-nm wavelength [Fig. 1(b)] with the quantum yield of $\sim 45 \%$ measured at 488-nm excitation with Rhodamine $6 \mathrm{G}$ as a reference dye.

Aqueous suspensions of the particles were prepared immediately before their use. Concentrations of UCPs and QDs were $0.625 \mu \mathrm{g} / \mu \mathrm{L}$ and $0.6 \times 10^{-6} \mathrm{~mol} / \mathrm{L}$, respectively. To prevent aggregation of the particles, UCP and QD suspensions were sonicated in an ultrasonic bath before their topical application to the skin surface.

\subsection{Experimental Methods}

\subsubsection{Particle delivery protocol}

All six rats were subjected to the procedure of FLMA. For carrying out the FLMA procedure, a commercially available Palomar Lux 2940 pulse erbium laser (Palomar Medical Technologies Inc., USA) operating at the 2940-nm wavelength was used. Laser pulse energy was $3 \mathrm{~J}$ with a duration of $20 \mathrm{~ms}$; the temporal profile of the pulse was modulated by three subpulses with duration of each about $5 \mathrm{~ms}$. The laser beam was split into 64 microbeams using a microlens array. Thus, 64 vertical microchannels were created over the $8 \times 8 \mathrm{~mm}^{2}$ skin area.

The animals were divided into two groups. In the first one (two animals), the efficiency of administration of UCP and QD suspensions was studied by using ultrasound or massage.

Five sites were marked as shown in Fig. 2(a): the site 0 corresponded to intact skin without any treatment, the sites 1 and 3 were treated by the UCP suspension, and the sites 2 and 4 by the QD suspension. The volume of both suspensions was equal to $23 \mu \mathrm{L}$. The UCPs and the QDs were delivered into microchannels by using sonophoresis (sites 3 and 4) or mechanical massage (sites 1 and 2) for 5 min each.

A commercially available device Dynatron 125 (Dynatronics, USA), equipped with a $2.2-\mathrm{cm}$ diameter US transducer was applied for sonophoresis in the continuous-wave mode with the frequency of $1 \mathrm{MHz}$ and the power density of $1.5 \mathrm{~W} / \mathrm{cm}^{2}$. Remains of the suspensions were removed from the skin surface using distilled water.

In the second group (four animals), six sites were marked as shown in Fig. 2(b): the site 0 corresponded to intact skin without any treatment, the sites 1 and 4 were treated by UCP suspension, and the sites 2 and 5 by the QD suspension. The volume of the applied suspensions was the same as for the first group. The site 3 remained untreated by any particle suspension. This site (control) was used for the monitoring of the changes of skin state after FLMA. The UCPs and the QDs were delivered into the microchannels by using sonophoresis (the sites 1, 2, 4, and 5) for $5 \mathrm{~min}$ each.

\subsubsection{Particle imaging}

Imaging of the particles was performed using different optical modalities, such as optical coherence tomography, luminescent spectroscopy, and confocal microscopy, as well as conventional optical microscopy (histological analysis).

The microchannels were visualized just after FLMA and after filling them by the particle suspensions in the first and the second groups. The imaging was performed with a commercially available spectral-domain optical coherence tomography (OCT) system OCP930SR (Thorlabs, USA), operating at the central wavelength of $930 \pm 5 \mathrm{~nm}$ with $100 \pm 5 \mathrm{~nm}$ fullwidth-at-half-maximum spectrum and having the following characteristics: numerical aperture of 0.22 , optical power of $2 \mathrm{~mW}$; the scanning length along the skin surface of $6 \mathrm{~mm}$ with the axial and the lateral resolutions on the air of 6.2 and $9.6 \mu \mathrm{m}$, respectively.

To study the influence of ultrasound and massage on intensity of luminescent signal from particles inside the skin, we carried out the spectral measurement immediately after FLMA and particle delivery in the first group of animals.

For monitoring UCP and QD localization in the rat skin in vivo (the second group), luminescent spectroscopy was applied every day during the entire period of in vivo studies (nine days). Figure 3 schematically shows the experimental system for detection of UCP and QD luminescent spectra by

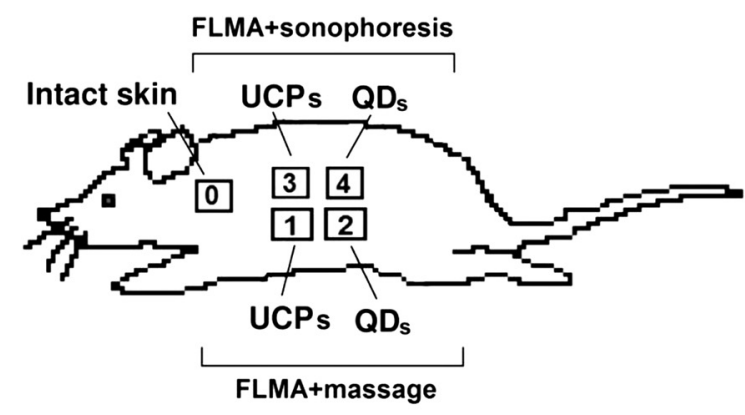

(a)

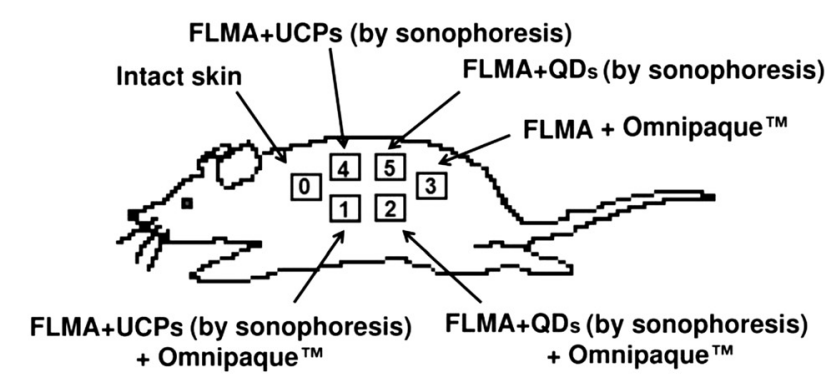

(b)

Fig. 2 Scheme of the studied sites of rat skin in vivo for (a) the first group and (b) the second group of experimental animals. 


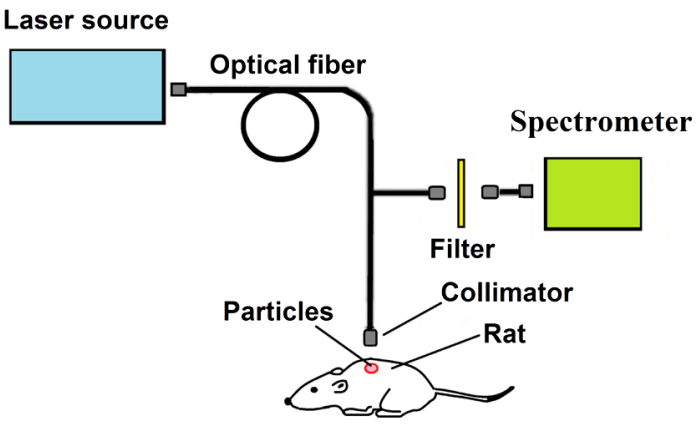

Fig. 3 Scheme of the experimental setup used for spatial localization of upconversion luminescent particles and QDs inside rat skin in vivo.

a spectrometer QE6500 (Ocean Optics, USA). The excitation of UCPs was performed at 980-nm wavelength and that of QDs at 405-nm using a semiconductor laser module DMH980-200 (Laser Systems LAS, Russia, $20 \mathrm{~mW}$ ) and a violet laser pointer Pen Style (HangZhou NaKu Technology Co. Ltd, China, $300 \mathrm{~mW}$ ), respectively. The fiber-optic probe was fixed over the skin surface at the distance of $25 \mathrm{~mm}$. Luminescent spectra were measured with a $100-\mathrm{ms}$ acquisition time.

The withdrawal of the animals from the experiment and sampling of tissues for the luminescent and morphological study was performed on the first day (two rats from the first and the second groups) and on the last day of the experiment (two rats from the second group).

Unstained sections were studies with luminescent microscopy. Since luminescence of UCPs and QDs is excited by different wavelengths, we used different equipment for the observation. Luminescent images of skin histological sections with QDs were obtained at room temperature $\left(\sim 20^{\circ} \mathrm{C}\right)$ using a standard confocal luminescent microscope (Leica TCS SP8, Germany) with $10 \times$ and $100 \times$ magnification under $405-\mathrm{nm}$ laser excitation. Images of UCPs were taken with an optical microscope OGME-PZ (Sapphire, Russia) equipped with a CCD camera (Videoscan 415/P-USB, Videoscan, Russia) under 980-nm laser excitation.

Then the sections were stained with hematoxylin-eosin according to the standard procedure. ${ }^{36}$ Morphometric studies of histological preparations were done using a digital image analysis system $\mu$ Vizo-103 medical microvisor (LOMO, Russia).

\subsubsection{Optical clearing agent}

To enhance the luminescent signal from the particles inside skin dermis, the skin sites 1,2 , and 3 were exposed during $20 \mathrm{~min}$ to OCA Omnipaque ${ }^{\mathrm{TM}}$ (GE Healthcare, Ireland) before each spectroscopic measurement.

Omnipaque $^{\mathrm{TM}}$ (Iohexol; GE Healthcare, Ireland) was used as an OCA to decrease skin scattering. Omnipaque ${ }^{\mathrm{TM}}$ is a nonionic, water-soluble $\mathrm{x}$-ray contrast agent with iodine concentration of $300 \mathrm{mg} / \mathrm{mL}$ commonly used for in vivo angiography and imaging of internal organs (arteriography, phlebography, etc.). Iohexol has a low osmolarity comparable with blood plasma and cerebral spinal fluid. It is approved by the U.S. Food and Drug Administration (FDA) for in vivo topical use. Omnipaque $^{\mathrm{TM}}$ (300) has molecular weight of 821 , neutral $\mathrm{pH}$ (6.8 to 7.6$)$, refractive index 1.439 at the wavelength $589 \mathrm{~nm}$, and low toxicity.
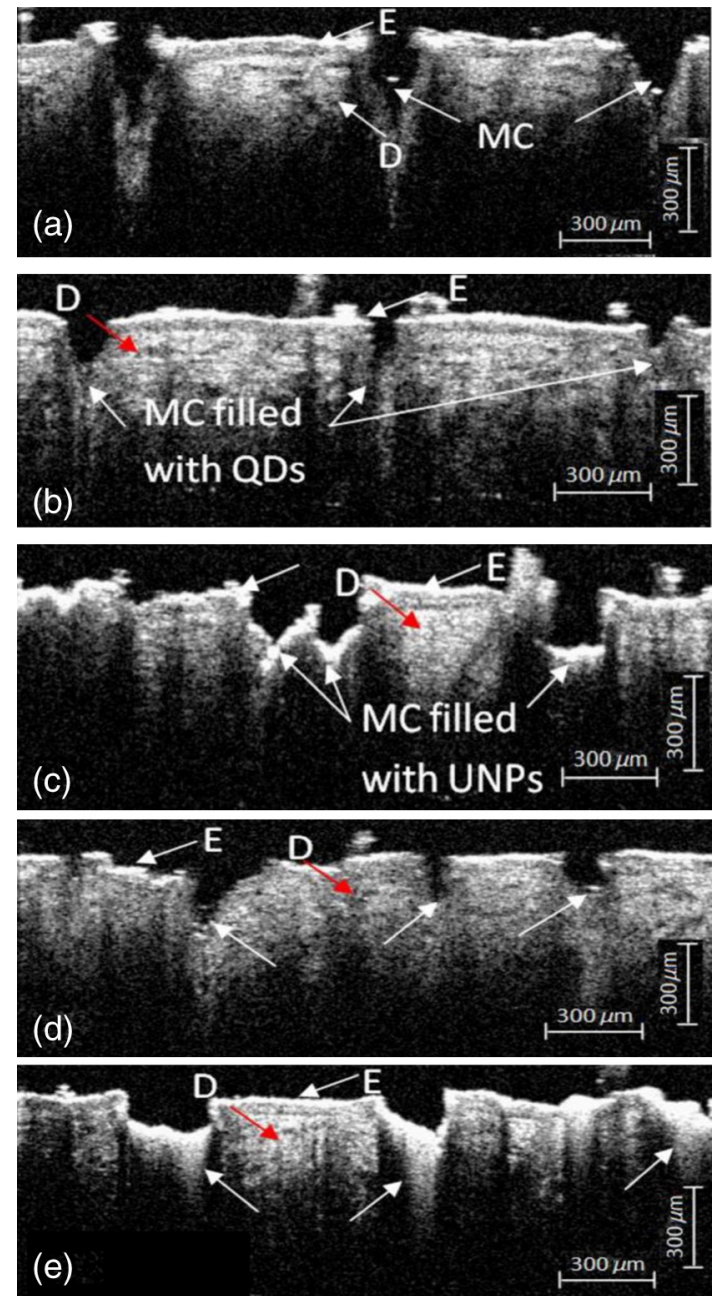

Fig. 4 The OCT images of FLMA-treated skin in vivo: (a) immediately after FLMA, (b) after QD delivery using a massage; (c) after UCP delivery using a massage; (d) after QD delivery using a sonophoresis, and (e) after UCP delivery using a sonophoresis. E, epidermis; $\mathrm{D}$, dermis; and MC, microchannel.

\section{Results and Discussion}

\subsection{Optical Coherence Tomography Imaging}

Figure 4 shows the series of OCT images of skin sites from the first group of rats immediately after FLMA alone (a), FLMA followed by QD delivery with massage (b), FLMA followed by UCP delivery with massage (c), FLMA followed by QD delivery with sonophoresis (d), and FLMA followed by UCP delivery with sonophoresis (e). In the images, two layersepidermis (E) and dermis (D)—are clearly distinguishable. Microchannels (MC) look like cones. Figure 4(a) allows for evaluation of the separation between the centers of the laserperforated channels as $\sim 1 \mathrm{~mm}$ with the diameter of their openings at skin surface as 150 to $250 \mu \mathrm{m}$ and the depth as 450 to $500 \mu \mathrm{m}$.

As follows from OCT images (c) and (e), after both massage and sonophoresis, the largest part of the microchannel is filled up by the UCP suspension. It is well seen that QDs cannot be visualized with high-contrast on the background of the skin scattering [see images (b) and (d)]. UCPs provide high contrast of OCT visualization of the microchannels because of their much 
larger size than QDs $(1.6 \mu \mathrm{m}$ versus $20 \mathrm{~nm})$. Comparison of data presented in Figs. 4(c) and 4(e) shows that in contrast to the massage, the ultrasound treatment allows for deeper filling of the ablated channels in the skin. It is in a good agreement with the results of Ref. 12, where the increase of skin permeability for both in vitro and in vivo studies using ultrasound irradiation was shown.

\subsection{Luminescent Analysis}

The spatial localization of the particles in the microchannels immediately after FLMA and the particles delivery can be observed on luminescent images presented in Fig. 5. Luminescence of UCPs was detected by optical microscope under 980-nm laser excitation [see Figs. 5(a) and 5(b)] and luminescent of QDs was detected by confocal microscope under 405-nm laser excitation [see Figs. 5(c) and 5(d)].

It is well seen that near the skin surface, the particles were localized along the channel walls; the main volume of the suspensions, apparently, was removed from the channels during the washing-off. However, as we can see in Fig. 4, the suspension remained at the depth of 400 to $500 \mu \mathrm{m}$. After healing of the tissue around the channels and as a result of their closing, the particles are accumulated within a localized space in tissue. QDs and UCPs were not detected in the histological sections with a confocal luminescent microscope after nine days of particle delivery.

Figure 6 shows the luminescent spectra of the UCPs [see Fig. 6(a)] and QDs delivered into skin by massage and sonophoresis [see Fig. 6(b)]. It can be seen that sonophoresis results in the increased luminescent signal from the particles in comparison with the massage, apparently, due to the increase of quantity of the particles penetrated into the channels.

Measured kinetics of luminescent intensity (Figs. 7 and 8) and histological analysis (Figs. 9 and 10) confirm that the depot of the particles can be created inside the microchannels.

Figure 7 shows the luminescent intensity produced by UCPs (a) and QDs (b) embedded into the rat skin in vivo.

Under excitation by $980-\mathrm{nm}$ laser, skin autofluorescence in the red spectral region is negligible. By contrast, 405-nm radiation excites fluorescence from endogenous skin fluorophores: three bands of porphyrins in the 630 to $710-\mathrm{nm}$ region and NADH near $480 \mathrm{~nm}^{37}$

The luminescent peak of QDs shifted in the skin from 680 to $670 \mathrm{~nm}$ overlaps with the fluorescent bands of porphyrins [see Fig. 7(b)]. The QD and UCP luminescence was observed for four days [see Fig. 7(b) $(3,4)$ ] and eight days [see Fig. 7(a) $(3,4)]$, respectively, with a gradual decrease in the intensity with time.
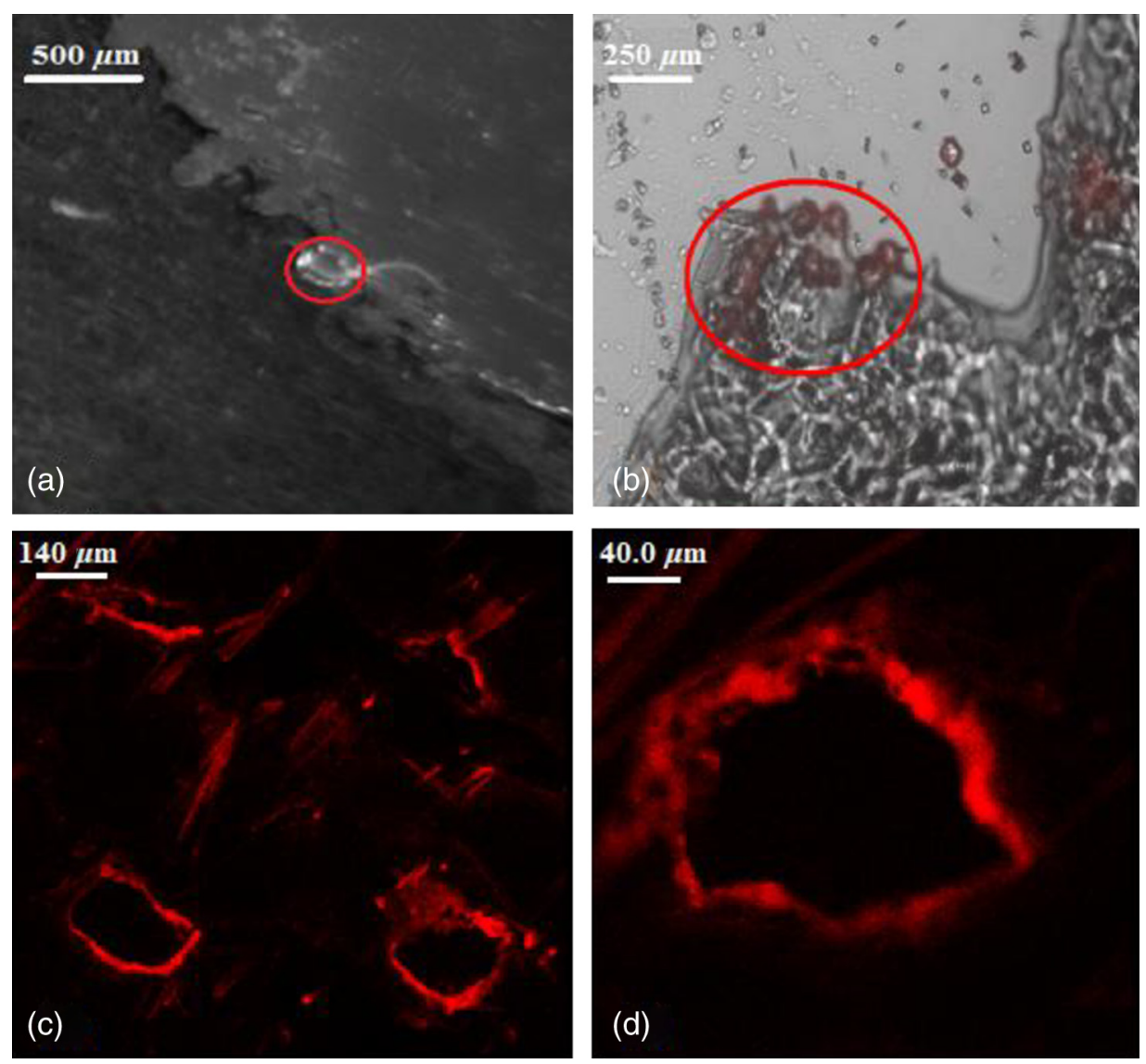

Fig. 5 Luminescent images of histological section of the perforated skin obtained using: $(a, b)$ an optical microscope equipped with CCD camera under 980-nm laser excitation of the UCPs and (c, d) a confocal microscope under 405-nm laser excitation of the QDs. The fluorescing UCPs are marked with the red circle. The images were obtained from the depth of (c) $0 \mu \mathrm{m}$ and (d) $40 \mu \mathrm{m}$ immediately after FLMA and particle delivery. 


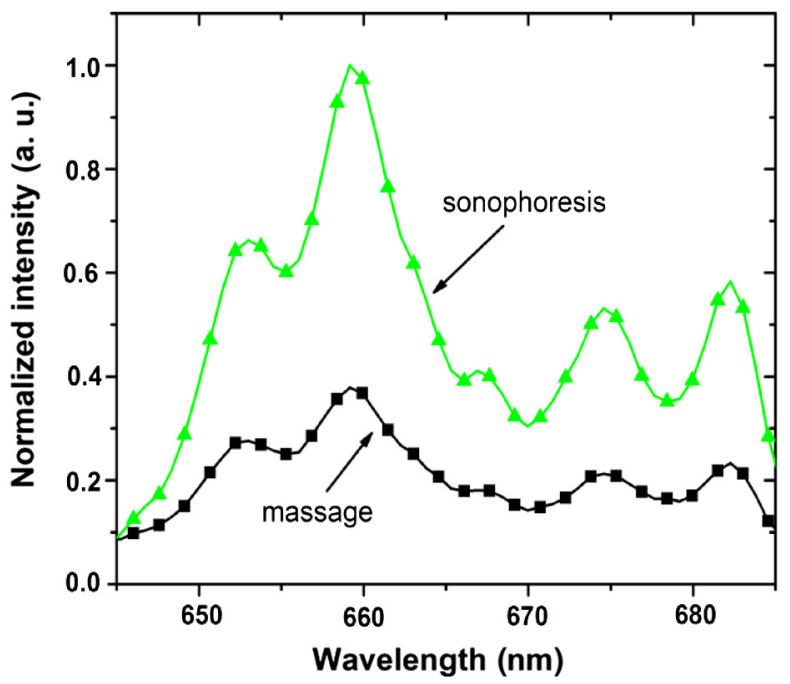

(a)

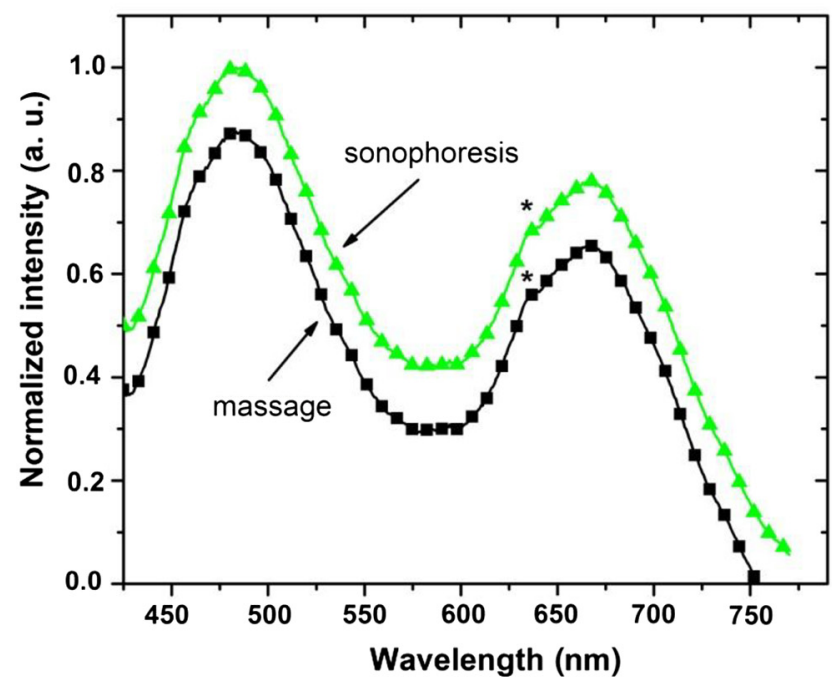

(b)

Fig. 6 Luminescent spectra of the (a) UCPs (excitation wavelength $980 \mathrm{~nm}$ ) and (b) QDs (excitation wavelength $405 \mathrm{~nm}$ ) delivered into skin by massage and by sonophoresis. Asterisk represents luminescent bands of endogenous porphyrins.

Healing and regeneration of skin epidermis (about 4 to 5 days) can prevent penetration of 405-nm light to the QD depot in the skin. At the same time, 980-nm light penetrates significantly deeper and can excite luminescence of UCPs for more prolonged time during skin healing and regeneration and afterward.

The decrease of luminescent signal can also be connected with the diffusion of the particles from the observation zone to surrounding tissues. In Ref. 38, it was shown that contrast of OCT-images of human skin with embedded particles also decreased during a few days after their delivery. After the full regeneration of the skin integrity, quantity of the particles was so little that they did not register in the OCT images. Difference between time interval (days) during which luminescence from UCPs and QDs is detectable can be related to particle size and their diffusion from the initial delivery volume: larger UCPs remained localized inside the skin channels for a long time, whereas much smaller QDs diffuse from the channels and distributed in the surrounding tissues.

It is well seen that the maximal luminescent signal is observed after the skin optical clearing. Figure 8 shows kinetics of the maximal intensity of the luminescent bands of the UCPs

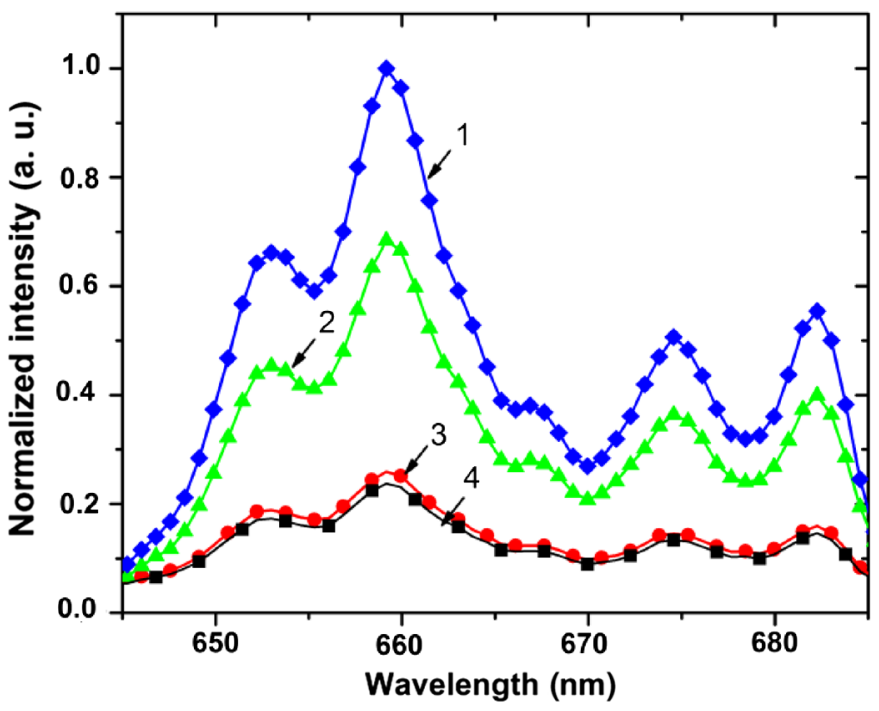

(a)

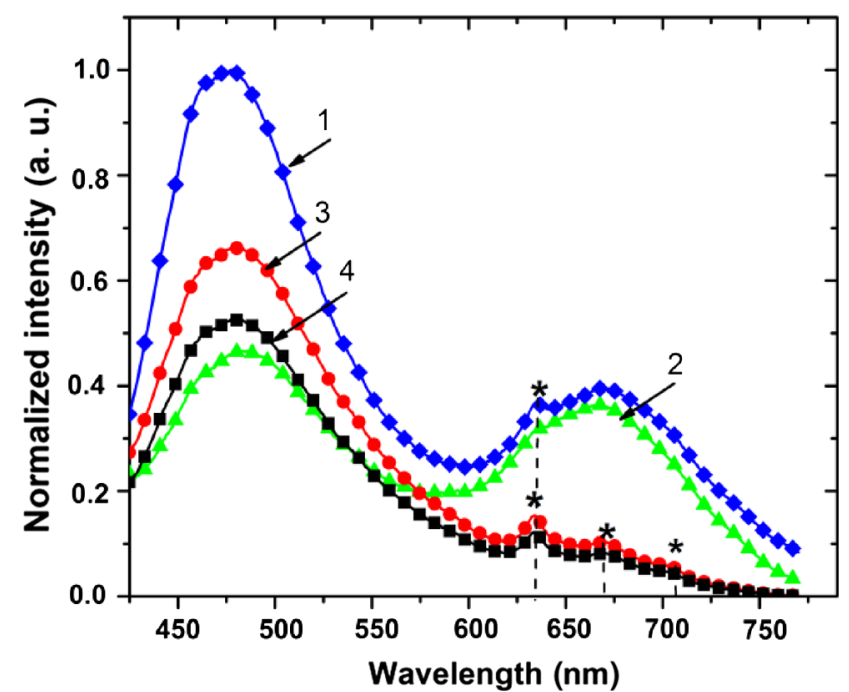

(b)

Fig. 7 Luminescent spectra of the (a) UCPs (excitation wavelength $980 \mathrm{~nm}$ ) and (b) QD (excitation wavelength $405 \mathrm{~nm}$ ) embedded into rat skin by sonophoresis: with topically applied Omnipaque ${ }^{\mathrm{TM}}$ immediately after FLMA and particle delivery (1); without Omnipaque ${ }^{\mathrm{TM}}$ immediately after FLMA and particle delivery (2); with Omnipaque ${ }^{\mathrm{TM}}$ after eight days of particle delivery (3); and without Omnipaque ${ }^{\mathrm{TM}}$ after eight days of particle delivery (4). Asterisk represents luminescent bands of endogenous porphyrins. 


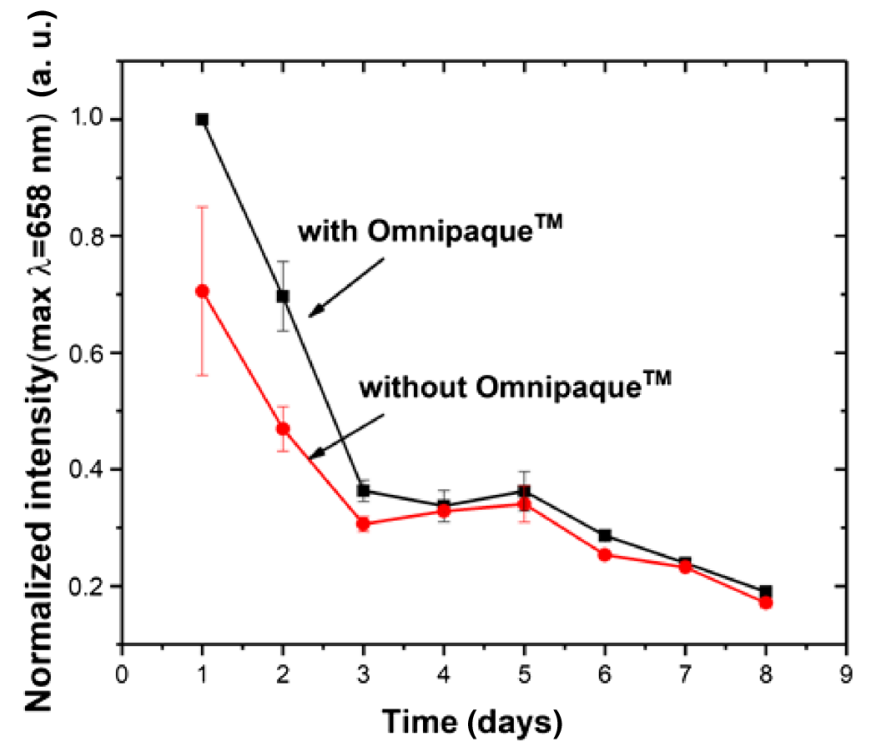

(a)

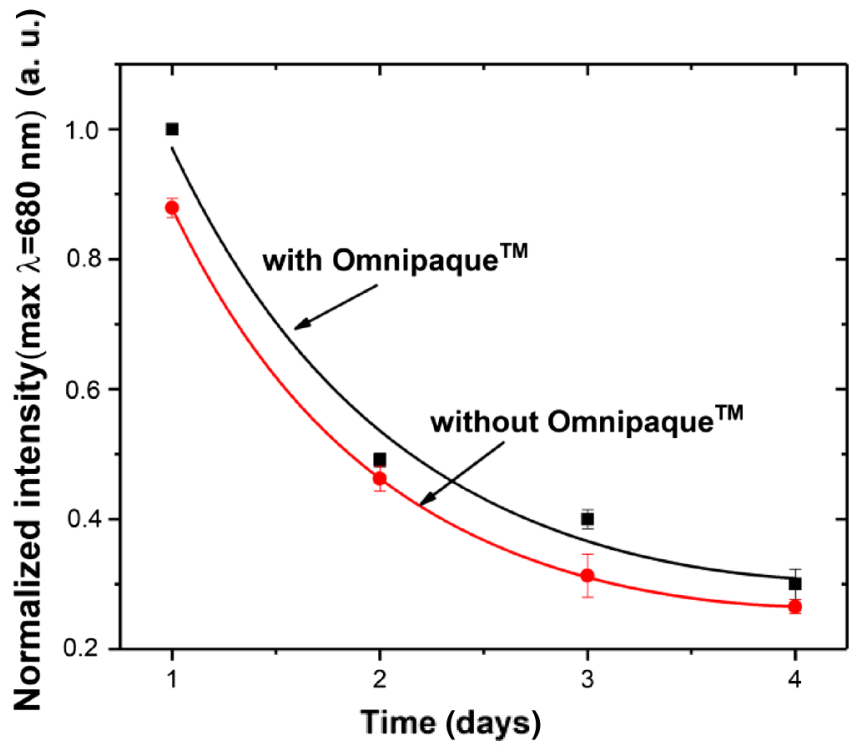

(b)

Fig. 8 The time dependence of the luminescent maximum of the (a) UCPs $\left(\lambda_{\max }=658 \mathrm{~nm}\right.$ ) and (b) QDs $\left(\lambda_{\max }=680 \mathrm{~nm}\right)$ embedded into the rat skin by sonophoresis and with (black squares) and without (red circles) optical clearing by topical application of Omnipaque ${ }^{\mathrm{TM}}$.

$\left(\lambda_{\max }=658 \mathrm{~nm}\right)(\mathrm{a})$ and QDs $\left(\lambda_{\max }=680 \mathrm{~nm}\right)(\mathrm{b})$ embedded into the rat skin by sonophoresis and with and without optical clearing by topical application of Omnipaque ${ }^{\mathrm{TM}}$.

Figures 7 and 8 show that the topical application of the immersion agent Omnipaque ${ }^{\mathrm{TM}}$ allows for enhancement of the luminescent signal from the particles located inside skin dermis due to reduction of light scattering of tissue layers, where excitation and fluorescent signals propagate. This is a so-called optical clearing technique application that increases probing depth and contrast of optical imaging methods. ${ }^{29}$ It was demonstrated, in particular, for fluorescent spectroscopy and microscopy. ${ }^{20,27,29}$ Omnipaque ${ }^{\mathrm{TM}}$ has refractive index (1.439) close to refractive index of main skin scatterers-collagen fibers (1.43). ${ }^{27}$ Thus, a partial replacement of the interstitial fluid by Omnipaque ${ }^{\mathrm{TM}}$ causes the matching of the refractive indices of the tissue components, its better homogeneity and reduction of scattering.

In Fig. 8(a), we can see that skin treatment by Omnipaque ${ }^{\mathrm{TM}}$ does not affect the intensity of the luminescent signal after four days post-FLMA and particle delivery. We suppose that after full regeneration of skin barrier function, Omnipaque ${ }^{\mathrm{TM}}$ does not penetrate effectively into skin dermis, and therefore, the 20-min agent application is not enough for signal enhancement.

\subsection{Histological Analysis}

Microphotographs of histological sections of the studied skin areas from the first group measured immediately after UCPs delivery are shown in Fig. 9. The control sample [intact skin without any treatment; see Fig. 9(a)] represents skin with normal multilayer epithelium (epidermis). The skin appendages are visible in the dermis. QDs could not be detected in the histological sections with conventional optical microscopy due to their small size $(20 \mathrm{~nm})$.

For the samples taken from the FLMA-zone [ablation only; see Fig. 9(b)], crater-like defects crossing epidermis and dermis, regularly occurring on average every $600 \mu \mathrm{m}$ with the development of coagulation necrosis in tissue around them, are observed. In Fig. 9(b), one of the craters covers an area of width $\times$ depth equal to $153 \times 170 \mu \mathrm{m}^{2}$ as the maximal depth of necrosis edge is $363 \mu \mathrm{m}$. Coagulation necrosis of the thickness about $10 \mu \mathrm{m}$ is formed around the perforated channel. In addition, full-blooded vessels around the zone of influence are observed.

Figure 9(c) shows UCPs on crater walls delivered by massage; this crater is an internal part of the microchannel. Outside, the channel is surrounded by a necrotic zone with a radius approximately equal to the crater depth. The size of the crater is $155 \times 58 \mu \mathrm{m}^{2}$.

It is important to note that sonophoresis increases the channel size. The form of the channel changes from conical to spherical. We anticipate that the necrotic tissue is detached and pushed out from the channel by sonophoresis. This hypothesis is confirmed by a thinner zone of coagulation necrosis observed in Fig. 9(d) than in Fig. 9(c).

Figure 10 presents a fragment of skin with a microchannel filled by UCPs of the rat from the second group. A depth of localization is $323 \mu \mathrm{m}$. The size of the defect is $21 \times 63 \mu \mathrm{m}^{2}$.

Analysis of this histological section confirms our hypothesis that particles with the microscale dimension localized inside the channels remain there after the channel healing and do not penetrate in surrounding tissue. Thus, a depot of particles can be created for prolonged drug release.

QDs, on the contrary, can diffuse through the surrounding tissue and leave the channel zone. Therefore, we cannot see accumulation of the particles in the histological sections and luminescent signal to the ninth day post-treatment. It was shown earlier that the use of FLMA allowed for delivery of microparticles into dermis and creation of a particle depot. ${ }^{11,38,39}$ For example, biocompatible $\mathrm{CaCO}_{3}$ microcontainers $(4.0 \pm 0.8 \mu \mathrm{m})$ containing $\mathrm{Fe}_{3} \mathrm{O}_{4}$ nanoparticles were delivered to the depth about $300 \mu \mathrm{m}$ in dermis. ${ }^{11}$ On the seventh 

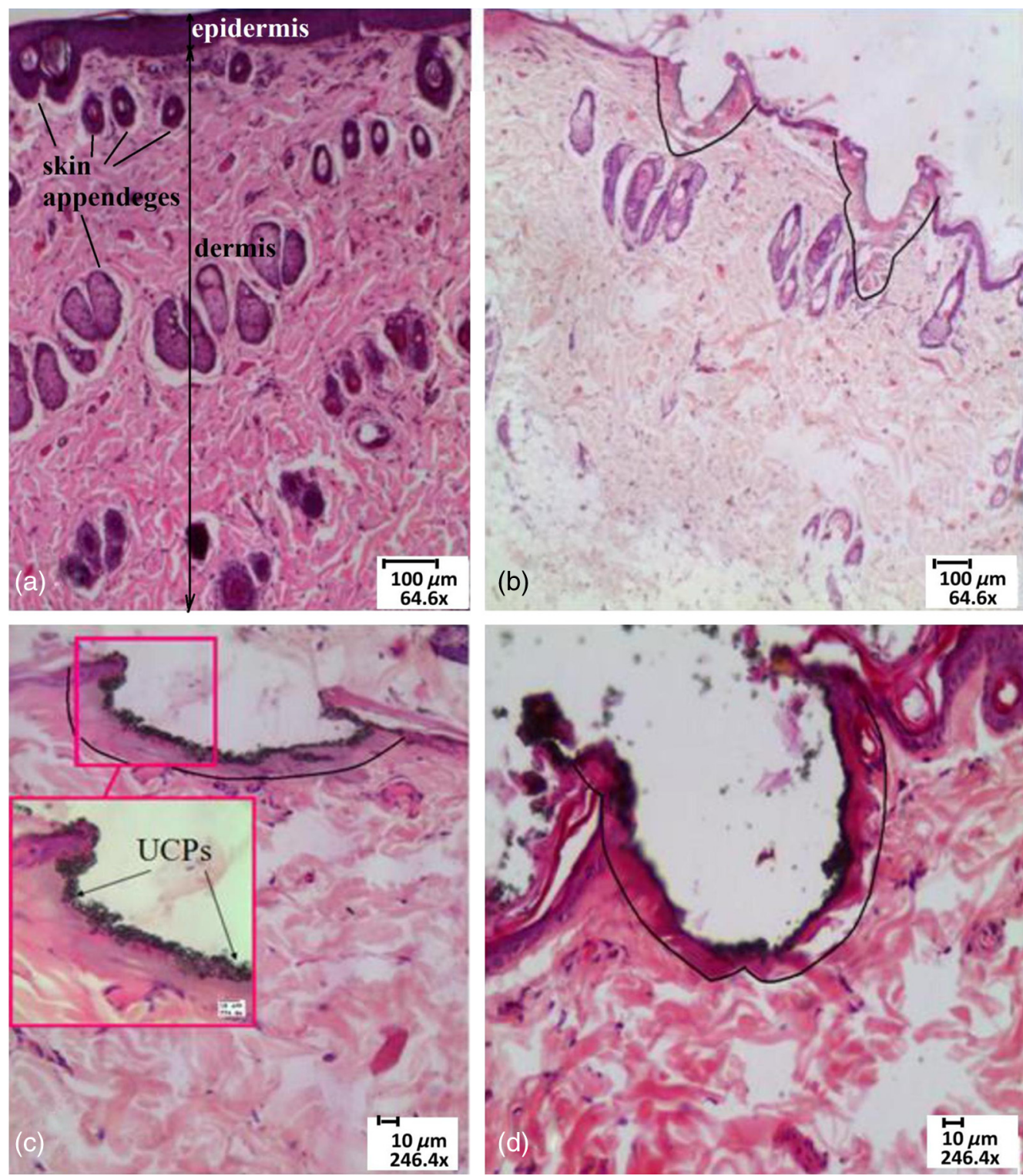

Fig. 9 Histological sections of the skin: (a) intact skin, (b) skin after laser ablation without embedded particles, (c) with the UCPs delivered by massage, and (d) with the UCPs delivered by sonophoresis (the mean size of the skin damaged area $[260 \pm 70] \times[110 \pm 30] \mu \mathrm{m}^{2}$ ). The samples were stained with hematoxylin and eosin. The contoured zones indicate coagulation necrosis (b, c, d).

day, the dissolving of the microcontainers and release of the content into dermis was observed. In Refs. 38 and 39, it was shown that particles with diameter about $100 \mathrm{~nm}$ and more can remain in skin in vivo during 30 days.

\section{Conclusions}

It is shown that UCPs are visualized in skin more efficiently in comparison with QDs. The UCPs have a stable narrow-band emission at excitation in the NIR spectral range. Under excitation by $980-\mathrm{nm}$ laser, the skin autofluorescence in the spectral region of the microparticle luminescence is absent. The luminescent images of the UCPs distribution indicate the advantage of the particle delivery into the skin by FLMA with sonophoresis. The UCPs are observed in the channels by optical coherence tomography, conventional microscopy, and luminescent imaging during eight days after the skin treatment. The QDs luminescence is detected for four days with a gradual decrease of the intensity. It is shown that the use of the immersion agent Omnipaque $^{\mathrm{TM}}$ results in the enhancement of the luminescent signal from the particles inside skin dermis. In the process of the channel healing, QDs were displaced from the channels to the surface of the skin. This fact explains the absence of the QD luminescence signal on the fifth day of the experiment. The UCPs were located deeper in the tissue and remained there after the healing of the channels. This explains the presence of the luminescent signal of the UCPs for eight days. The difference in the depth of the particles localization can be explained by the fact that ultrasound more effectively influences heavier and larger UCPs than QDs.

Thus, the multimodal imaging approach provides capabilities for a more advanced investigation of spatial localization of 


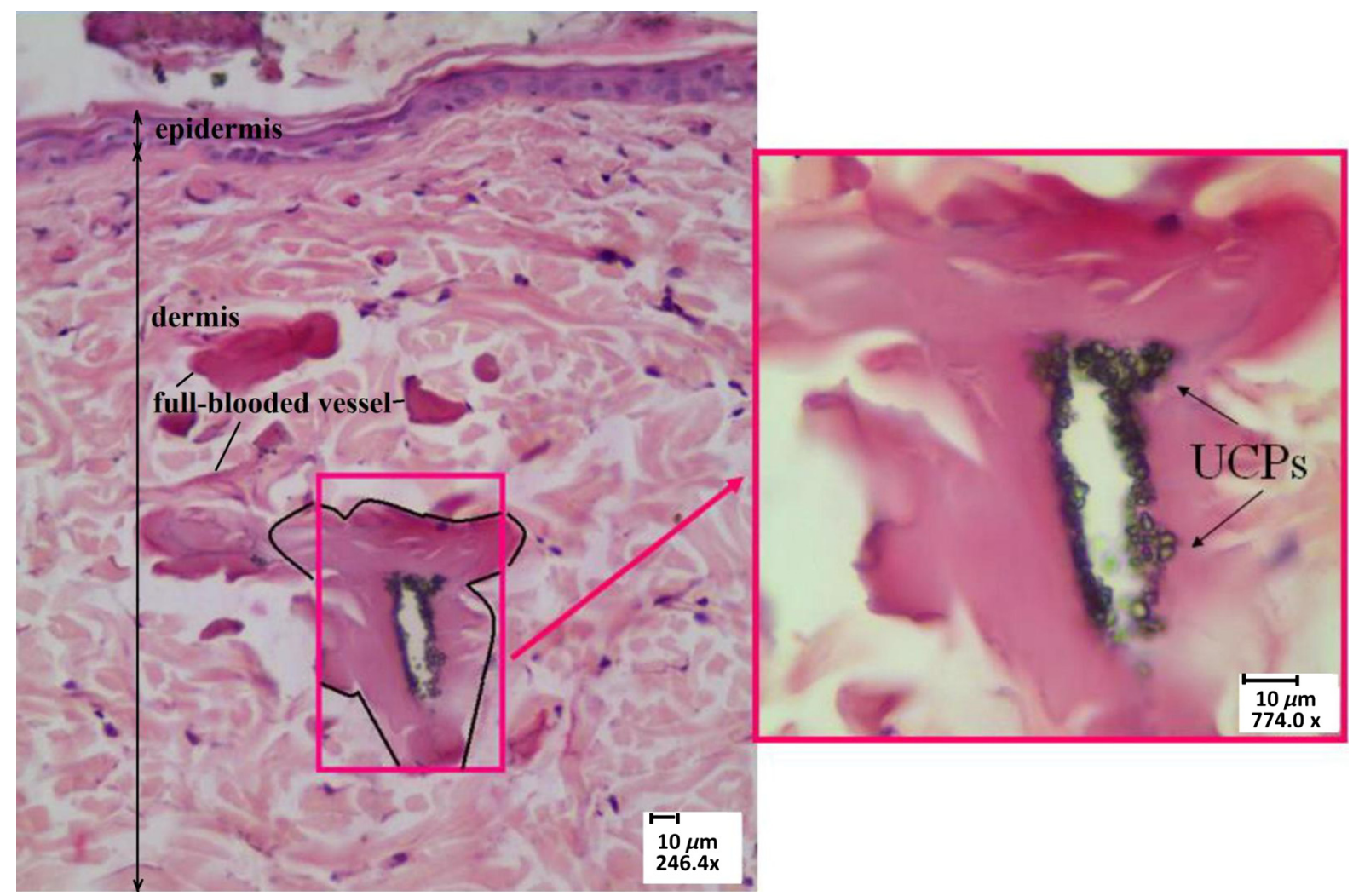

Fig. 10 Histological sections of the perforated skin with the UCPs delivered by sonophoresis after nine days. The samples were stained with hematoxylin and eosin. The contoured zones indicate coagulation necrosis.

UCPs and QDs within the skin, which is of interest for different biomedical applications.

\section{Appendix: CulnS $/$ /ZnS Core/Shell Synthesis}

A $\mathrm{Zn}$ precursor was prepared by mixing 1.6-g zinc stearate $\mathrm{Zn}(\mathrm{St})_{2}$ (2.53 mmol), 4.3-mL oleic acid, $14-\mathrm{mL}$ octadecene in a flask and flushing the mixture for 30 min with nitrogen, followed by heating up to $190^{\circ} \mathrm{C}$ until a colorless solution was obtained..$^{35}$ Then the flask was cooled down to $90^{\circ} \mathrm{C}$, $0.84 \mathrm{~mL}$ dodecanethiol was added and the mixture was left under nitrogen at $75^{\circ} \mathrm{C}$ for further use. For the core/shell synthesis, the flask with $\mathrm{CuInS}$ cores grown at $220^{\circ} \mathrm{C}$ was cooled down to $80^{\circ} \mathrm{C}$. At this temperature, $2.7 \mathrm{~mL}$ of the $\mathrm{Zn}$ precursor $(\mathrm{Zn}: \mathrm{In}=1: 1)$ was added and the flask was immediately heated up to $215^{\circ} \mathrm{C}$ and was kept at this temperature for $30 \mathrm{~min}$. Afterward, the flask was cooled down again to $80^{\circ} \mathrm{C}, 2.7 \mathrm{~mL}$ of the $\mathrm{Zn}$ precursor $(2.7 \mathrm{ml})$ was added followed by heating up the flask again up to $215^{\circ} \mathrm{C}$. The same procedure was repeated five times. Finally, the solution was cooled down, diluted with chloroform $\left[\mathrm{V}\left(\mathrm{CHCl}_{3}\right): \mathrm{V}(\right.$ solution $\left.)=1: 5\right]$ and centrifuged at $3000 \mathrm{~g}$ for $10 \mathrm{~min}$. The formed precipitate was discarded, and ethanol was added to the supernatant until opalescence. ${ }^{35}$ The mixture was centrifuged at $3000 \mathrm{~g}$ for $10 \mathrm{~min}$ and the supernatant was decanted. The precipitate was dispersed in toluene. This precipitation/dissolution procedure was repeated three times and in such a way obtained QDs were stored in toluene at $4^{\circ} \mathrm{C} .{ }^{35}$

\section{Disclosures}

All authors have no financial interest.

\section{Acknowledgments}

This study was supported by Academy of Finland (288164 EKV, IYuY; 260321, 290596, APP); E.K.V. was also supported by the Russian Governmental contract 2014/203, Project 1490; E.A.G., A.B.B., N.A.N., G.B.S., and V.V.T. were supported by the Russian Governmental grant 14. Z50.31.0004; I. Yu Y. and E.K.V. were supported by the Russian Presidential grant MK-6009.2016.2; E.K.V., I. Yu Y., A.N.B., V.I.K., A.P.P., I.V.M., and V.V.T. acknowledge support by the Russian Governmental grant 074-U01; E.K.V., I. Yu Y., E.A.G., A.N.B., J.G.K., V.I.K., and V.V.T. were supported by the Russian Presidential grant NSh-7898.2016.2.

\section{References}

1. H. Schaefer and T. E. Redelmeier, "Prediction and measurement of percutaneous absorption," in Skin Barrier: Principles of Percutaneous Absorption, H. Schaefer and T. E. Redelmeier, Eds., pp. 118-152, Karger, Basel (1996).

2. J. Lademann et al., "Penetration of titanium dioxide microparticles in a sunscreen formulation into the horny layer and the follicular orifice," Skin Pharmacol. Appl. Skin Phys. 12(5), 247-256 (1999).

3. S. E. Cross et al., "Human skin penetration of sunscreen nanoparticles: in-vitro assessment of a novel micronized zinc oxide formulation," Skin Pharmacol. Physiol. 20(3), 148-154 (2007).

4. R. Toll et al., "Penetration profile of microspheres in follicular targeting of terminal hair follicles," J. Invest. Dermatol. 123(1), 168-176 (2004).

5. A. K. Kohli and H. O. Alpar, "Potential use of nanoparticles for transcutaneous vaccine delivery: effect of particle size and charge," Int. J. Pharm. 275(1-2), 13-17 (2004).

6. J. Lademann et al., "Nanoparticles - an efficient carrier for drug delivery into the hair follicles," Eur. J. Pharm. Biopharm. 66(2), 159-164 (2007). 
7. S. E. Lee et al., "Penetration pathways induced by low-frequency sonophoresis with physical and chemical enhancers: iron oxide nanoparticles versus Lanthanum Nitrates," J. Invest. Dermatol. 130(4), 1063-1072 (2010).

8. I. Lavon et al., "Bubble growth within the skin by rectified diffusion might play a significant role in sonophoresis," J. Controlled Release 117(2), 246-255 (2007).

9. G. Cevc and U. Vierl, "Nanotechnology and the transdermal route. A state of the art review and critical appraisal," J. Controlled Release 141(3), 277-299 (2010)

10. I. A. Aljuffali, C.-H. Lin, and J.-Y. Fang, "Skin ablation by physical techniques for enhancing dermal/transdermal drug delivery," J. Drug Delivery Sci. Technol. 24(3), 277-287 (2014).

11. E. A. Genina et al., "Optical monitoring of transcutaneous delivery of composite microparticles in vivo," Biomed. Opt. Express 7(6), 2082-2087 (2016).

12. G. S. Terentyuk et al., "Use of fractional laser microablation and ultrasound to facilitate the delivery of gold nanoparticles into skin in vivo," Quantum Electron. 42(6), 471-477 (2012).

13. H.-J. Laubach et al., "Skin responses to fractional photothermolysis," Lasers Surg. Med. 38(2), 142-149 (2006).

14. E. Moczko et al., "Dyes assay for measuring physicochemical parameters," J. Anal. Chem. 81(6), 2311-2316 (2009).

15. E. Moczko et al., "Optical assay for biotechnology and clinical diagnosis," IEEE Trans. Biomed. Eng. 58(8), 2154-2160 (2011).

16. X. Michalet et al., "Quantum dots for live cells, in vivo imaging, and diagnostics," Science 307, 538-544 (2005).

17. E. A. Grebenik et al., "Feasibility study of the optical imaging of a breast cancer lesion labeled with upconversion nanoparticle biocomplexes," J. Biomed. Opt. 18(7), 076004 (2013).

18. N. R. Popova et al., "Layer-by-layer capsules as smart delivery systems of $\mathrm{CeO}_{2}$ nanoparticle-based theranostic agents," Nanosystems $8(2)$, 282-289 (2017).

19. M. Yao et al., "Multifunctional $\mathrm{Bi}_{2} \mathrm{~S}_{3} /$ PLGA nanocapsule for combined HIFU/radiation therapy," Biomaterials 35(28), 8197-8205 (2014).

20. A. P. Popov et al., "Enhancement of upconversion deep-tissue imaging using optical clearing," Proc. SPIE 9540, 95400B (2015).

21. R. Li et al., "Enhancing the imaging and biosafety of upconversion nanoparticles through phosphonate coating," ACS Nano 9(3), 32933306 (2015).

22. L. Tang et al., "In vivo skin penetration and metabolic path of quantum dots," Sci. China Life Sci. 56(2), 181-188 (2013).

23. F. Wang, Y. Chen, and H. A. E. Benson, "Formulation of nano and micro PLGA particles of the model peptide insulin: preparation, characterization, stability and deposition in human skin," Open Drug Delivery J. 2, 1-9 (2008).

24. X. Michalet et al., "Quantum dots for live cells, in vivo imaging, and diagnostics," Science 307(5709), 538-544 (2005).

25. N. Zhou, J. Ni, and R. He, "Advances of upconversion nanoparticles for molecular imaging," Nano Biomed. Eng. 5(3), 131-139 (2013).

26. A. Sadovoy et al., "Micro-encapsulated bio-markers for assessment of stress conditions in aquatic organisms in vivo," Laser Phys. Lett. 9(7), 542-546 (2012).

27. V. V. Tuchin, Tissue Optics: Light Scattering Methods and Instruments for Medical Diagnostics, 3rd ed., Vol. PM 254, p. 988, SPIE Press, Bellingham, Washington (2015).

28. E. V. Khaydukov et al., "Enhanced spatial resolution in optical imaging of biotissues labelled with upconversion nanoparticles using a fibreoptic scanning technique," Laser Phys. Lett. 11(9), 095602 (2014).

29. E. A. Genina et al., "Optical clearing of biological tissues: prospects of application in medical diagnostics and phototherapy [Review]," J. Biomed. Photonics Eng. 1(1), 22-58 (2015).

30. D. W. Straughan, "First European Commission report on statistic of animal use," Altern. Lab. Anim. 22, 289-292 (1994).

31. G. Chen et al., "Upconversion nanoparticles: design, nanochemistry, and applications in theranostics," Chem. Rev. 114(10), 5161-5214 (2014).

32. F. Zhang, Photon Upconversion Nanomaterials, Nanostructure Science Technology, pp. 1-413, Springer, New York (2015).

33. F. Auzel, "Upconversion and anti-stokes processes with $\mathrm{f}$ and $\mathrm{d}$ ions in solids," Chem. Rev. 104(1), 139-174 (2004).

34. D. J. Gargas et al., "Engineering bright sub-10-nm upconverting nanocrystals for single-molecule imaging," Nat. Nanotechnol. 9, 300-305 (2014).
35. E. S. Speranskaya et al., "Hydrophilic, bright $\mathrm{CuInS}_{2}$ quantum dots as Cd-free fluorescent labels in quantitative immunoassay," Langmuir 30(25), 7567-7575 (2014).

36. F. L. Carson and C. C. Hladik, Histotechnology: A Self-Instructional Text, 4th ed., pp. 118-120, ASCP Press, Chicago (2015).

37. V. V. Dremin and A. V. Dunaev, "How the melanin concentration in the skin affects the fluorescence-spectroscopy signal formation," J. Opt. Technol. 83(1), 43-48 (2016).

38. E. A. Genina et al., "Transcutaneous delivery of micro- and nanoparticles with laser microporation," J. Biomed. Opt. 18(11), 111406 (2013).

39. E. A. Genina et al., "Fractional laser microablation of skin aimed at enhancing its permeability for nanoparticles," Quantum Electron. 41(5), 396-401 (2011).

Elena K. Volkova received her $\mathrm{PhD}$ in biophysics from Saratov State University (SSU), Saratov, Russia, in 2013. She has authored 14 papers in peer-reviewed journals and 12 papers in conference proceedings. From 2012 to 2018, she was a research associate of Laboratory of Biomedical Optics of Research-Educational Institute of Optics and Biophotonics at SSU. Since 2018, she is an engineer at the Research and Education Institution of Nanostructures and Biosystems at Saratov State University. Her research interests include the absorption and luminescence spectroscopy of nanoparticles and crystals, study of methods for the synthesis of nanoparticles, nanoparticles for biological applications, visualization of the structure of biological tissue by upconversion nanoparticles optics of biological tissues, biomedical optics, optical control parameters of biological tissues, spectroscopy and imaging in biomedicine, physics, optical, and laser measurements.

Irina Yu Yanina received her PhD degree in biophysics from Saratov State University (SSU), Saratov, Russia, in 2013. She has authored 17 papers in peer-reviewed journals and 18 papers in conference proceedings. From 2010 to present, she is an associate fellow researcher of Laboratory of Biomedical Optics of Research-Educational Institute of Optics and Biophotonics at SSU. Her research interests include the development of optical methods of fat tissue destructive engineering, photodynamic/photothermal therapy, biomedical optics, drug delivery, spectroscopy and imaging in biomedicine, optical, and laser measurements.

Elina A. Genina is an associate professor in the Departments of Optics and Biophotonics of Saratov State University. She received her PhD in biophysics from Saratov State University, Saratov, Russia, in 2002. She has authored more than 200 peer-reviewed publications and 7 book chapters on biomedical optics. She is a reviewer and a guest-editor of peer-reviewed journals, such as J. Biomed. Opt., J. Innovative Optical Health Sciences, Quantum Electronics, Optics and Spectroscopy, etc. She is a scientific secretary of International School for junior scientists and students on Optics, Laser Physics and Biophotonics (Saratov Fall Meeting). Her research interests include biomedical optics, laser medicine, and development of methods for control of tissue absorption and scattering properties for medical optical diagnostics and therapy.

Alexey N. Bashkatov received his $\mathrm{PhD}$ in biophysics from Saratov State University (SSU), Saratov, Russia, in 2002. He has authored 200 invited and contributed publications, 7 book chapters, and over 250 invited and contributed conference presentations. From 2002 to present, he is an associate professor in the Departments of Optics and Biophotonics of Saratov State University. $\mathrm{He}$ is a head of Laboratory of Biomedical Optics of Research-Educational Institute of Optics and Biophotonics at SSU and senior researcher of Interdisciplinary Laboratory of Biophotonics at Tomsk State University. His current research interests include study of optical properties of tissues, spectroscopy, development of methods for control of tissue absorption and scattering properties for medical optical diagnostics and therapy, and modeling of light propagation in turbid media.

Julia G. Konyukhova is a senior engineer of the Optics and Biophotonics Department at Saratov State University (National Research University of Russia). She received her PhD in physics and mathematics from Saratov State University in 1999. Her research interests include physics and chemistry of solids, light-emitting nanomaterials, 
spectroscopy, luminescence, photochemistry, and methods of structural analysis. She is a coauthor of over 40 papers in peer-reviewed scientific journals and international conference proceedings.

Alexey P. Popov graduated with honors from Physics Department of M.V. Lomonosov Moscow State University (MSU), Russia, in 2003, and his PhD from MSU in 2006 and his DSc (Tech.) degree from the University of Oulu, Finland, in 2008. Currently, he is an adjunct professor at the University of Oulu. His scientific interests are in the area of nanobiophotonics, light-biotissue interaction, optical imaging and sensing, and design of biotissue-mimicking phantoms. He has published over 100 journal papers and peer-reviewed conference proceedings; organized such international conferences as Advanced Laser Technologies ALT-2007 (Levi, Finland), Laser Applications in Life Sciences LALS-2010 (Oulu, Finland), and Summer SPIE FOCUS School on Optics and Photonics 2017 (Oulu, Finland). He served as a guest editor of the Journal of Biophotonics (Special Issue devoted to the LALS-2010 conference). He is a SPIE senior member, a SPIE student chapter faculty advisor, and an OSA member.

Elena S. Speranskaya received her $\mathrm{PhD}$ in chemistry at Saratov State University in 2013. In 2013 to 2014, she worked at Ghent University as a postdoc. Her research interests include synthesis of different quantum dots and its use as fluorescent biolabels in several applications. Currently she is a researcher at Saratov State University.

Alla B. Bucharskaya received her PhD from 2010 to present. She is a head of Centre of Collective Use of Saratov State Medical University n.a. V.I. Razumovsky. The author of 5 patents for inventions and more than 20 papers in peer-reviewed journals. She is a member of Russian Association of Clinical Cytologists. Her research interests include cytology, morphology, oncology, photodynamic/photothermal therapy, biomedical optics, and nanomedicine.

Nikita A. Navolokin graduated from the Saratov State Medical University n.a. V.I. Razumovsky in 2013. Since 2014, he is an assistant in the Department of Pathological Anatomy and researcher in Centre of Collective Use of Saratov State Medical University. He is an author of 5 patents for inventions and more than 80 scientific publications. His sphere of scientific interests is morphology, oncology, photodynamic/ photothermal therapy, biomedical optics, and drug delivery.

Irina Yu. Goryacheva graduated from Saratov State University as chemist in 1993. She obtained her PhD in analytical chemistry in
1999 and DSc in 2007. Her research interests involve development of luminescent semiconductor quantum dots for immunoassay and bioassay, synthesis and studying of luminescent carbon nanoparticles, and new formats of immunoassay and sensors.

Vyacheslav I. Kochubey is a professor of optics and biophotonics at Saratov National Research State University. His research interests include spectroscopy, tissue optics, nanoparticles, and nanobiophotonics. He has published more than 150 peer-reviewed scientific papers.

Gleb B. Sukhorukov holds the chair in biopolymers at the School of Engineering and Materials Science, Queen Mary University of London (QMUL). He graduated from the Department of Physics, Lomonosov Moscow State University and after postdoctoral positions at academia and industry. He started his independent research group at Max Planck Institute of Colloids and Interfaces in 2001 with help of Sofja Kovalevskaja Award of Alexander von Humboldt Foundation until he moved to QMUL in 2006. He pioneered research on layerby-layer assembled polyelectrolyte capsules with most research outcome relevant to development of multifunctional drug delivery systems enabling encapsulation of various substances in capsules of defined size with triggered release induced by light, magnetic field, and ultrasound. He was named among the top-10 world known scientists of Russian origin by Forbes.

Igor V. Meglinski is a professor at the Opto-Electronics and Measurement Techniques research unit in the University of Oulu (Finland). His research interests include propagation of coherent polarized light in turbid tissue-like scattering media, coherent effects of multiple scattering of light, tissue polarimetry, angular momentum of light, optical phase singularities, dynamic light scattering, Monte Carlo modeling and multimodal imaging. $\mathrm{He}$ is a chartered physicist (CPhys), fellow of the Institute of Physics (London, UK), senior member of IEEE, and fellow of SPIE.

Valery V. Tuchin is a professor and head of optics and biophotonics at Saratov National Research State University and several other universities and institutions. His research interests include tissue optics, laser medicine, tissue optical clearing, and nanobiophotonics. He is a fellow of SPIE and OSA. He has been awarded Honored Science Worker of Russia, SPIE Educator Award, FiDiPro (Finland), Chime Bell Prize of Hubei Province (China), and Joseph W. Goodman Book Writing Award (OSA/SPIE). 